\title{
Adaptive Dynamic Surface Control for Generator Excitation Control System
}

\author{
Zhang Xiu-yu, ${ }^{1,2}$ Liu Cui-ping, ${ }^{1}$ Wang Jian-guo, ${ }^{1}$ and Lin Yan ${ }^{2}$ \\ ${ }^{1}$ Northeast Dianli University, School of Automation, Jilin City, Jilin 132012, China \\ ${ }^{2}$ Beijing University of Aeronautics and Astronautics, School of Automation, Beijing 100191, China \\ Correspondence should be addressed to Zhang Xiu-yu; zhangxiuyu80@163.com
}

Received 7 April 2014; Revised 14 June 2014; Accepted 18 June 2014; Published 7 July 2014

Academic Editor: Qingsong Xu

Copyright (c) 2014 Zhang Xiu-yu et al. This is an open access article distributed under the Creative Commons Attribution License, which permits unrestricted use, distribution, and reproduction in any medium, provided the original work is properly cited.

\begin{abstract}
For the generator excitation control system which is equipped with static var compensator (SVC) and unknown parameters, a novel adaptive dynamic surface control scheme is proposed based on neural network and tracking error transformed function with the following features: (1) the transformation of the excitation generator model to the linear systems is omitted; (2) the prespecified performance of the tracking error can be guaranteed by combining with the tracking error transformed function; (3) the computational burden is greatly reduced by estimating the norm of the weighted vector of neural network instead of the weighted vector itself; therefore, it is more suitable for the real time control; and (4) the explosion of complicity problem inherent in the backstepping control can be eliminated. It is proved that the new scheme can make the system semiglobally uniformly ultimately bounded. Simulation results show the effectiveness of this control scheme.
\end{abstract}

\section{Introduction}

With the development of power system, the requirements of the load are increasing. However, the ability of the transmission network is limited and the electric power system has gradually reached its operating limit; the world power systems tend to extend power grid scale and develop larger power system. However, the power systems are more likely to encounter some problems such as oscillations and complex nonlinear phenomena due to the extension of power grid scale and some emergencies. Therefore, ways of maintaining the reliability and stability of power system are attracting more and more research interest [1-4]. Moreover, the promotion of the control ability of the generator excitation system can help us to monitor the states of system and predict system performance; it is the most effective way to improve the performance of the whole excitation control system and it has been widely of concern in recent years [5-7].

The most common method for designing generator excitation system controller is the direct-feedback-linearization, which transforms the nonlinear model into a linear one by some mathematical ways. However, it is only effective to the system when special conditions are satisfied $[8,9]$. Now, more and more nonlinear control techniques are being applied to the generator excitation systems. In [10], an output feedback controller combined with high-order sliding-mode techniques is proposed to guarantee the transient stability of the power systems. In [11], for the nonlinear characteristics of the transient process in power system whose parameters are known, Colbia-Vega and his collaborators designed a controller for steam-valving and excitation system, which can stabilize the voltage and improve power quality. In [12], an adaptive fuzzy zero dynamic and sliding model control is proposed to cope with the unknown parameters, and the fuzzy logic rules are used to approximate the unknown nonlinear functions. For the time-varying and uncertainties of the reactance and voltage in one-machine infinite-bus power system, Zhang and Zhu in [13] designed an adaptive excitation controller by using strong tracking filter in the design procedure of excitation controller of generators, and the stability of the control system is improved when it is accompanied with some disturbances; however, it has the explosion of complicity problem inherent in the backstepping control. In [14], an adaptive filter control for generator excitation system was proposed based on IEEE 14-bus. 


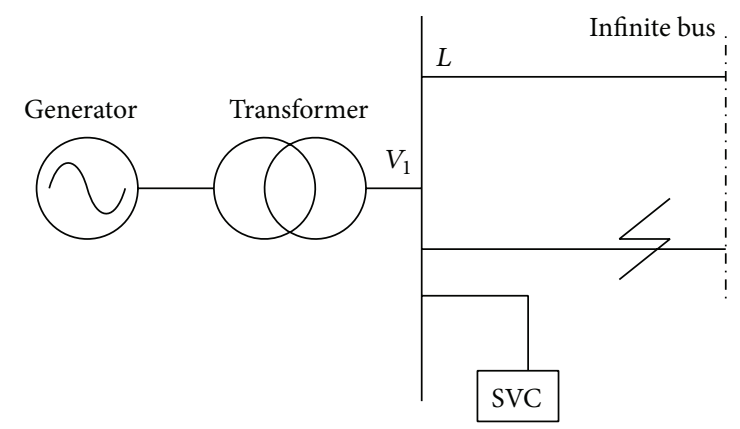

FIgURE 1: Single machine infinite bus system with SVC.

Motivated by the previous work $([12,13]$, etc.) this paper has successfully fused adaptive dynamic surface control with neural network and tracking error transformed function to design a robust adaptive controller for the nonlinear power system. The features of our control scheme are as follows:

(i) the tracking error of the power angle and voltage at the SVC can be transformed to an arbitrarily prespecified performance by an error transformed function;

(ii) the neural network based adaptive dynamic surface controller is designed without doing any linearization to the excitation generator model. Therefore, the design procedures and the final control law are greatly simplified and the control accuracy is improved;

(iii) by using the neural network, the structure and the parameters of the control system can be totally unknown;

(iv) by estimating the norm of the weighted vector of neural network instead of the weighted vector itself, the computational burden is greatly reduced and it is more suitable for the real time control.

This paper is organized as follows. In Section 2, the mathematical model of excitation system of power generator is introduced and the control purpose is formulated. In Section 3, the design procedure of the neural network and the tracking error transformed function based adaptive DSC scheme is presented. Section 4 gives the stability analysis for the proposed scheme. Finally, simulation examples are given to demonstrate the effectiveness of the proposed design method.

\section{The Excitation System of Power Generator}

2.1. Mathematical Model. Assume that the SVC device is installed in a single-machine infinite-bus system; the structure and the equivalent circuit diagram are shown in Figure 1 [15].

To proceed, the following assumptions are made.

Assumption 1. Ignoring the dynamic process of rapid excitation equipment means the control voltage $u_{f}$ is equal to the generator excitation voltage $E_{f}, E_{f}=u_{f}$.
Assumption 2. The mechanical power of generators remains unchanged in transient stability process; that is, $\Delta P_{m}=0$.

Then, the mathematical model of the generator excitation control system equipped with SVC can be described as follows [16-18]:

$$
\begin{aligned}
& \dot{\delta}=\omega-\omega_{0}, \\
& \dot{\omega}=\frac{\omega_{0}}{H} P_{m 0}-\frac{D}{H}\left(\omega-\omega_{0}\right)-\frac{\omega_{0}}{H} \frac{E_{q}^{\prime} V_{s}}{X_{d \Sigma}^{\prime}} \sin \delta+d_{1}, \\
& \dot{E}_{q}^{\prime}=-\frac{X_{d \Sigma}}{T_{d 0} X_{d \Sigma}^{\prime}} E_{q}^{\prime}+\frac{1}{T_{d 0}} \frac{X_{d}-X_{d}^{\prime}}{X_{d \Sigma}^{\prime}} V_{s} \cos \delta+\frac{1}{T_{d 0}} u_{f}+d_{2}, \\
& \dot{B}_{L}=\frac{1}{T_{C}}\left(-B_{L}+B_{C}+u_{B}\right),
\end{aligned}
$$

where $\delta$ is the power angle of the generator running rotor; $\omega$ is the rotor speed of the generator $(\mathrm{rad} / \mathrm{s}) ; D$ is the damping constant; $H$ is the inertia constant; $\omega_{0}=2 \pi f_{0}$ is the synchronous machine speed $(\mathrm{rad} / \mathrm{s}) ; f_{0}$ is the rated frequency $(\mathrm{Hz}) ; P_{m}$ is the mechanical power of motor output; $T_{d 0}$ is the direct axis transient short circuit time constant (s); $T_{C}$ is the time constant of adjusting system and SVC (s); $E_{q}^{\prime}$ is the transient electromotive force (EMF) of the generator; $V_{s}=1$ is the voltage of the system; $u_{f}$ is the control voltage of excitation equipment; $u_{B}$ is the input of SVC; $B_{L}$ is the adjustable equivalent susceptance in SVC; $B_{C}$ is the initial value of adjustable susceptance; $d_{1}$ is position bounded torque interference of the rotor; and $d_{2}$ is electromagnetic transient interference of the stator:

$$
\begin{aligned}
X_{1} & =X_{d}^{\prime}+X_{T}, \\
X_{3} & =X_{d}+X_{T}, \\
X_{d \Sigma} & =X_{3}+X_{2}+X_{3} X_{2}\left(B_{L}-B_{C}\right), \\
X_{d \Sigma}^{\prime} & =X_{1}+X_{2}+X_{1} X_{2}\left(B_{L}-B_{C}\right),
\end{aligned}
$$

where $X_{2}$ is the transmission line reactance; $X_{d}$ is the generator direct axis reactance; $X_{d}^{\prime}$ is the direct axis transient reactance of the generator; and $X_{T}$ is the transformer reactance.

When the system encounters the external disturbances, the stability of the power angle and SVC access point voltage can be effectively improved by combining the regulation of SVC with generator-excitation adjustment. In this paper, we take increment of the power angle and accessing point voltage as the output value of the system to design our adaptive dynamic surface controller; that is,

$$
\begin{aligned}
& y_{1}=\delta-\delta_{0}, \\
& y_{2}=V_{m}-V_{\text {ref }},
\end{aligned}
$$


where $V_{m}$ is the accessing point voltage and $V_{\text {ref }}$ is the reference voltage. According to the circuit, we have

$$
V_{m}=\frac{\sqrt{\left(X_{2} E_{q}^{\prime}\right)^{2}+\left(X_{1} V_{s}\right)^{2}+2 X_{1} X_{2} E_{q}^{\prime} V_{s} \cos \delta}}{X_{d \Sigma}^{\prime}} .
$$

Based on the above descriptions, let $x_{1}=\delta-\delta_{0}, x_{2}=$ $\omega-\omega_{0}, x_{3}=E_{q}^{\prime}$, and $x_{4}=V_{m}-V_{\text {ref }}$; then, (1) can be expressed as the following form:

$$
\begin{gathered}
\dot{x}_{1}=x_{2}, \\
\dot{x}_{2}=-\frac{\omega_{0}}{H} \frac{V_{s} \sin \left(x_{1}+\delta_{0}\right)}{X_{d \Sigma}^{\prime}} x_{3}-\frac{D}{H} x_{2} \\
+\frac{\omega_{0}}{H} P_{m 0}+d_{1}, \\
\dot{x}_{3}=\frac{1}{T_{d 0}} u_{f}-\frac{X_{d \Sigma}}{T_{d 0} X_{d \Sigma}^{\prime}} x_{3}+\frac{1}{T_{d 0}} \frac{X_{d}-X_{d}^{\prime}}{X_{d \Sigma}^{\prime}} V_{s} \cos \delta+d_{2}, \\
\dot{x}_{4}=-\frac{V_{s} \sin \delta X_{1} X_{2}}{V_{m}\left(X_{d \Sigma}^{\prime}\right)^{2}} x_{2}-\frac{X_{1} X_{2} V_{m}}{X_{d \Sigma}^{\prime}} \cdot \frac{1}{T_{c}}\left(-B_{L}+B_{C}\right) \\
+\frac{X_{2}^{2} x_{3}+X_{1} X_{2} V_{s} \cos \delta}{V_{m}\left(X_{d \Sigma}^{\prime}\right)^{2}} \\
+\left(-\frac{X_{d \Sigma}}{T_{d 0} X_{d \Sigma}^{\prime}} x_{3}+\frac{1}{T_{d 0}} \frac{X_{d}-X_{d}^{\prime}}{X_{d \Sigma}^{\prime}} \cdot V_{s} \cos \delta\right) \\
+\frac{u_{f}}{T_{d 0} V_{m}\left(X_{d \Sigma}^{\prime}\right)^{2}} \cdot\left(X_{2}^{2} x_{3}+X_{1} X_{2} V_{s} \cos \delta\right) \\
+\frac{X_{1} X_{2}}{T_{C} X_{d \Sigma}^{\prime}}\left(-x_{4} u_{B}\right) .
\end{gathered}
$$

Let

$$
\begin{gathered}
g_{2}=-\frac{\omega_{0}}{H} \frac{V_{s} \sin \left(x_{1}+\delta_{0}\right)}{X_{d \Sigma}^{\prime}}, \quad g_{3}=\frac{1}{T_{d 0}}, \\
g_{4}=\frac{X_{1} X_{2}}{T_{C} X_{d \Sigma}^{\prime}}, \quad f_{2}\left(\bar{x}_{2}\right)=-\frac{D}{H} x_{2}+\frac{\omega_{0}}{H} P_{m 0}, \\
u_{B}^{\prime}=-x_{4} u_{B}, \\
\cdot \frac{1}{T_{c}}\left(-B_{L}+B_{C}\right)+\frac{X_{2}^{2} x_{3}+X_{1} X_{2} V_{s} \cos \delta}{V_{m}\left(X_{d \Sigma}^{\prime}\right)^{2}} \\
\cdot\left(-\frac{V_{m}\left(X_{d \Sigma}^{\prime}\right)^{2}}{T_{d 0} X_{d \Sigma}^{\prime}} x_{3}-\frac{X_{1} X_{2} V_{m}}{X_{d \Sigma}^{\prime}}\right. \\
\left.+\frac{X_{2}^{2} x_{3}+X_{1} X_{2} V_{s} \cos \delta}{T_{d 0} V_{m}\left(X_{d \Sigma}^{\prime}\right)^{2}} \cdot \frac{X_{d}-X_{d}^{\prime}}{X_{d \Sigma}^{\prime}} V_{s} \cos \delta\right) \\
f_{3}\left(\bar{x}_{3}\right)=-\frac{X_{d \Sigma}}{T_{d 0} X_{d \Sigma}^{\prime}} x_{3}+\frac{1}{T_{d 0}} \frac{X_{d}-X_{d}^{\prime}}{X_{d \Sigma}^{\prime}} V_{s} \cos \delta,
\end{gathered}
$$

where $\bar{x}_{i}:=\left[x_{1}, \ldots, x_{i}\right]^{T} \in R^{i}, i=1, \ldots, 4$, are state vectors. Then, the system (5) can be converted into the following two independent subsystems (7) and (8), whose relative degree is 3 and 1 , respectively:

$$
\begin{aligned}
& \dot{x}_{1}=x_{2}, \\
& \dot{x}_{2}=g_{2} x_{3}+f_{2}\left(\bar{x}_{2}\right)+d_{1}, \\
& \dot{x}_{3}=g_{3} u_{f}+f_{3}\left(\bar{x}_{3}\right)+d_{2}, \\
& y_{1}=x_{1}, \\
& \dot{x}_{4}=g_{4} u_{B}^{\prime}+f_{4}\left(\bar{x}_{4}\right), \\
& y_{2}=x_{4} .
\end{aligned}
$$

Now, we can design our adaptive dynamic surface control law of the power system based on the following assumptions.

Assumption 3. The value of $g_{2}$ is unknown but the sign of $g_{2}$ is known; it is assumed that there exist two constants $g_{m}$ and $g_{2 \max }$ satisfying

$$
0<g_{m} \leq\left|g_{2}\right| \leq g_{2 \max }
$$

Assumption 4. The reference signal $y_{r}$ is smooth and bounded; $\left[y_{r}, \dot{y}_{r}, \ddot{y}_{r}\right]^{T}$ belong to a compact set for all $t \geq 0$.

Remark 5. Assumptions 1 and 2 which are the same as those in [15] are used to construct the model of the excitation system of power generator. Assumption 3 is used in Section 4, such as in the proof of (59), (65), and (67). We also emphasize that $g_{m}$ and $g_{2 \max }$ are not required in the implementation of our control law but are required for analysis only. Assumption 4 is common in all dynamic surface control schemes.

2.2. RBF Neural Network. In general, the neural network is a multi-input single-output system [19]; the mathematical expression is

$$
Y=\vartheta^{T} \psi(\xi)
$$

where $\xi \in \mathbb{R}^{n}$ is the input of RBF neural network; $Y \in \mathbb{R}$ is the output of RBFNN, $\vartheta \in \mathbb{R}^{N}$ is an $N$-dimensional parameter vector, and here $N$ is the number of neurons; $\psi(\xi): \mathbb{R}^{n} \rightarrow$ $\mathbb{R}^{N}$ is nonlinear vector function and $\psi(\xi)=\left[\psi_{1}(\xi), \ldots\right.$, $\left.\psi_{N}(\xi)\right]^{T}$ and $\psi_{k}(\xi)$ is the Gauss function.

Lemma 6. RBFNN is a universal approximator in the sense that, given any real continuous function $\mathscr{F}(\cdot): \Omega_{\xi} \rightarrow \mathbb{R}$ with $\Omega_{\xi} \subseteq \mathbb{R}^{n}$ a compact set and any $\delta_{m}>0$, by appropriately choosing $\sigma_{k}$ and $\zeta_{k}, k=1, \ldots, N$, for some sufficiently large integer $N$, there exists an RBF network [20], $\forall \xi \in \Omega_{\xi} \subseteq \mathbb{R}^{n}$, $|\delta| \leq \delta_{m}$,

$$
\mathscr{F}(\xi)=\mathcal{\vartheta}^{* T} \psi(\xi)+\delta(\xi)
$$


where $\vartheta^{*}$ is the optimal weight vector of $\vartheta$ and $\delta(\xi)$ is approximation error and can be defined as

$$
\begin{gathered}
\vartheta^{*}=\underset{\vartheta \in \mathbb{R}^{n}}{\arg \min }\left\{\sup _{\xi \in \Omega_{\xi}}|Y(\xi)-\mathscr{F}(\xi)|\right\}, \\
\delta(\xi)=\mathscr{F}(\xi)-\vartheta^{* T} \psi(\xi) .
\end{gathered}
$$

2.3. Error Transformed Function. We define the tracking error:

$$
e:=y-y_{r},
$$

where $y_{r}$ is the desired trajectory. According to [21, 22], the performance function and error transformed function are defined as follows: a performance function $\varrho(t): \mathbb{R}_{+} \rightarrow$ $\mathbb{R}_{-}-\{0\}$ is defined as a positive smooth decreasing function, such that, for all $t \geq 0$,

$$
\begin{array}{ll}
-\sigma \omega(t)<e(t)<\omega(t), & \text { if } e(0)>0, \\
-\varpi(t)<e(t)<\sigma \omega(t), & \text { if } e(0)<0,
\end{array}
$$

where $0<\sigma<1$ and $\lim _{t \rightarrow \infty}=\omega_{\infty}>0$ and $\omega_{\infty}$ is the maximum allowable tracking error of steady-state. To convert (14) into an equivalent unconstrained one, define the error transformed function

$$
e(t):=\omega(t) \Phi\left(S_{1}\right),
$$

where $S_{1}$ is the transformed error and $\Phi\left(S_{1}\right)$ is a smooth and strictly monotone increasing function; the inverse function has the following properties:

$$
\begin{array}{ll}
-\sigma<\Phi\left(S_{1}\right)<1, & \text { if } e(0)>0, \\
-1<\Phi\left(S_{1}\right)<\sigma, & \text { if } e(0)<0,
\end{array}
$$

where

$$
\begin{array}{lll}
\lim _{S_{1} \rightarrow-\infty} \Phi\left(S_{1}\right)=-\sigma, & \lim _{S_{1} \rightarrow \infty} \Phi\left(S_{1}\right)=1, & \text { if } e(0)>0, \\
\lim _{S_{1} \rightarrow-\infty} \Phi\left(S_{1}\right)=-1, & \lim _{S_{1} \rightarrow \infty} \Phi\left(S_{1}\right)=\sigma, & \text { if } e(0)<0 .
\end{array}
$$

As (17) shows, if $S_{1} \in \mathscr{L}_{\infty}$, (16) holds. Take $\varpi(t)>$ 0 and (15) into consideration, and we can have $-\sigma \omega(t)<$ $\varpi(t) \Phi\left(S_{1}\right)<1$ (if $e(0)>0$ ) or $-\varpi(t)<\omega(t) \Phi\left(S_{1}\right)<\sigma \omega(t)$ (if $e(0)<0)$; that is, (14) holds. Hence, from the above analysis, to achieve the prespecified tracking performance, we just only need to show that $S_{1} \in \mathscr{L}_{\infty}$, where the strictly increasing property of $\Phi\left(S_{1}\right)$ guarantees that we can obtain

$$
S_{1}=\Phi^{-1}\left(\frac{e(t)}{\omega(t)}\right) .
$$

Note that the case $e(0)=0$ can be incorporated into $e(0)>0$ or $e(0)<0$. At the same time, $\sigma$ cannot be chosen to be zero due to $S_{1}(0)$ being infinite.

\section{Adaptive Dynamic Surface Control of Generator Excitation System}

According to the mathematical model of the generator excitation system that is shown in (7), we design adaptive dynamic surface controller about power angle subsystem as follows.

Step 1. Let the first surface error and the tracking error as (18) be defined:

$$
\begin{gathered}
\dot{S}_{1}=\Psi\left[-\frac{\dot{\omega}}{\omega} e+x_{2}-\dot{y}_{r}\right], \\
\Psi:=\frac{1}{\omega} \frac{\partial \Phi^{-1}}{\partial(e / \omega)},
\end{gathered}
$$

where $y_{r}$ is an ideal output of the power angle; we suppose that $y_{r}$ is a smooth and continuous function, $e:=y_{1}-y_{r}=$ $x_{1}-y_{r}$.

Consider the following quadratic function:

$$
V_{1}=\frac{1}{2} S_{1}^{2} \text {. }
$$

We can obtain that

$$
\dot{V}_{1}=S_{1}\left[\Psi\left(-\frac{\dot{\omega}}{\omega} e-\dot{y}_{r}\right)+\Psi\left(x_{2}-x_{2 d}\right)+\Psi x_{2 d}\right],
$$

where $x_{2 d}$ is the virtual control signal that will be designed; choose the virtual control law as follows to stabilize (21):

$$
x_{2 d}=\frac{\left[-k_{1} S_{1}-\Psi\left(-(\dot{\omega} / \varpi) e+\Psi S_{1}-\dot{y}_{r}\right)\right]}{\Psi} .
$$

In order to avoid "differential explosion," the new variable $z_{2}$ is obtained by first-order low pass filter:

$$
\tau_{2} \dot{z}_{2}+z_{2}=x_{2 d}
$$

where $\tau_{2}$ is the filter time constant.

Step 2. Define the second surface error:

$$
S_{2}=x_{2}-z_{2} .
$$

Consider the following quadratic function:

$$
V_{2}=\frac{1}{2}\left(S_{2}^{2}+\frac{g_{m}}{\gamma_{v_{2}}} \widetilde{v}_{2}^{2}\right)
$$

The time derivative of $V_{2}$ is

$$
\dot{V}_{2}=S_{2}\left[g_{2} x_{3}+f_{2}\left(\bar{x}_{2}\right)+d_{1}-\dot{z}_{2}\right]+\frac{g_{m}}{\gamma_{v_{2}}} \widetilde{v}_{2} \dot{\hat{v}}_{2} .
$$

We need to use neural network to approximate the unknown terms on a compact set $\Omega_{\xi_{2}}$ :

$$
\frac{1}{g_{2}}\left[f_{2}\left(\bar{x}_{2}\right)-\dot{z}_{2}+d_{1}+\frac{3}{2} g_{2}^{2} S_{2}\right]=\theta_{\delta_{2}}^{* T} \psi_{2}\left(\xi_{2}\right)+\delta_{2}^{*}\left(\xi_{2}\right),
$$


where $\xi_{2}:=\left(x_{1}, x_{2}, x_{2 d}, z_{2}\right) \in \Omega_{\xi_{2}} \subset \mathbb{R}^{4}$; define $\widetilde{v}_{2}=\widehat{v}_{2}-v_{2}^{*}$, and $\gamma_{v_{2}}$ is a positive design parameter. Let the estimated value of $v_{2}^{*}=\left(1 / g_{m}\right)\left\|\theta_{\delta_{2}}^{* T}\right\|^{2}$ be $\widehat{v}_{2}$, according to inequality

$$
\begin{aligned}
g_{2} S_{2} \theta_{\delta_{2}}^{* T} \psi_{2} & \leq \frac{g_{m} \alpha_{2}^{2} S_{2}^{2} v_{2}^{*} \psi_{2}^{T} \psi_{2}}{2}+\frac{g_{2 \max }^{2}}{2 \alpha_{2}^{2}}, \\
g_{2} S_{2} \delta_{2} & \leq \frac{1}{2} g_{2}^{2} S_{2}^{2}+\frac{1}{2} \delta_{2}^{* 2}\left(\xi_{2}\right) .
\end{aligned}
$$

From (27) and (28), we have

$$
\begin{aligned}
\dot{V}_{2} \leq & S_{2}\left[g_{2}\left(x_{3}-x_{3 d}\right)+g_{2} x_{3 d}+\frac{g_{m} \alpha_{2}^{2} S_{2} v_{2}^{*} \psi_{2}^{T} \psi_{2}}{2}\right] \\
& -g_{2}^{2} S_{2}^{2}+\frac{1}{2} \delta_{2}^{* 2}+\frac{g_{2 \max }^{2}}{2 \alpha_{2}^{2}}+\frac{g_{m}}{\gamma_{v_{2}}} \widetilde{v}_{2} \dot{\hat{v}}_{2},
\end{aligned}
$$

where $x_{3 d}$ is the virtual control signal to be designed; let the power angle actual control law and parameter adjustment law, respectively, be as follows:

$$
\begin{gathered}
x_{3 d}=\operatorname{sgn}\left(g_{2}\right)\left(-k_{2} S_{2}-\frac{\alpha_{2}^{2} S_{2} \widehat{v}_{2} \psi_{2}^{T} \psi_{2}}{2}\right), \\
\dot{\hat{v}}_{2}=\gamma_{v_{2}}\left[\frac{\alpha_{2}^{2} S_{2}^{2} \psi_{2}^{T} \psi_{2}}{2}-\sigma_{v_{2}} \widehat{v}_{2}\right] .
\end{gathered}
$$

The new variable $z_{3}$ is obtained by first-order low pass filter:

$$
\tau_{3} \dot{z}_{3}+z_{3}=x_{3 d}
$$

where $\tau_{3}$ is the filter time constant. In order to avoid "differential explosion" problem brought by backstepping control, we use $z_{3}$ instead of $x_{3 d}$ in the following.

Step 3. Define the third surface error:

$$
S_{3}=x_{3}-z_{3} \text {. }
$$

Consider the following quadratic function:

$$
V_{3}=\frac{1}{2}\left(\frac{1}{g_{3}} S_{3}^{2}+\frac{1}{\gamma_{v_{3}}} \widetilde{v}_{3}^{2}\right) \text {. }
$$

The time derivative of $V_{3}$ is

$$
\dot{V}_{3}=S_{3}\left[u_{f}+\frac{f_{3}\left(\bar{x}_{3}\right)}{g_{3}}+\frac{d_{2}}{g_{3}}-\frac{\dot{z}_{3}}{g_{3}}\right]+\frac{1}{\gamma_{v_{3}}} \widetilde{v}_{3} \dot{\widehat{v}}_{3} .
$$

Similar to second step, we use neural network to approximate the unknown term in a compact set:

$$
\frac{1}{g_{3}}\left[f_{3}\left(\bar{x}_{3}\right)-\dot{z}_{3}+d_{2}\right]=\theta_{\delta_{3}}^{* T} \psi_{3}\left(\xi_{3}\right)+\delta_{2}^{*}\left(\xi_{3}\right),
$$

where $\xi_{3}:=\left(x_{1}, x_{2}, x_{3}, x_{3 d}, z_{3}\right) \in \Omega_{\xi_{3}} \subset \mathbb{R}^{5}$; let $\widetilde{v}_{3}=\widehat{v}_{3}-v_{3}^{*}$, $\gamma_{v_{3}}$ is positive design parameter, and $\widehat{v}_{3}$ is the estimate value of $v_{3}^{*}=\left\|\theta_{\delta_{3}}^{* T}\right\|^{2}$. Consider the hypothesis; we have

$$
\begin{aligned}
\dot{V}_{3} \leq & S_{3}\left[u_{f}+\frac{\alpha_{3}^{2} S_{3} v_{3}^{*} \psi_{3}^{T} \psi_{3}}{2}+\frac{S_{3}}{2}\right] \\
& +\frac{1}{2} \delta_{3}^{* 2}\left(\xi_{3}\right)+\frac{1}{2 \alpha_{3}^{2}}+\frac{1}{\gamma_{v_{3}}} \widetilde{v}_{3} \dot{\hat{v}}_{3} .
\end{aligned}
$$

Let the power angle actual control law and parameter adjustment law be, respectively, as follows:

$$
\begin{aligned}
& u_{f}=-k_{3} S_{3}-\frac{\alpha_{3}^{2} S_{3} \widehat{v}_{3} \psi_{3}^{T} \psi_{3}}{2}-\frac{S_{3}}{2}, \\
& \dot{\hat{v}}_{3}=\gamma_{v_{3}}\left[\frac{\alpha_{3}^{2} S_{3}^{2} \psi_{3}^{T} \psi_{3}}{2}-\sigma_{v_{3}} \widehat{v}_{3}\right] .
\end{aligned}
$$

Similarly, we design controller for the voltage subsystem (8); surface error is defined in (18):

$$
\begin{gathered}
\dot{S}_{4}=\Psi_{2}\left[-\frac{\dot{\omega}_{2}}{\omega_{2}} e_{2}+g_{4} u_{B}^{\prime}+f_{4}\left(\bar{x}_{4}\right)-\dot{y}_{r 2}\right], \\
\Psi_{2}:=\frac{1}{\omega_{2}} \frac{\partial \Phi^{-1}}{\partial\left(e_{2} / \omega_{2}\right)},
\end{gathered}
$$

where $e_{2}:=y_{2}-y_{r 2}=x_{4}-y_{r 2}, y_{r 2}$ is the ideal voltage reference signal; consider the following quadratic function:

$$
V_{4}=\frac{1}{2}\left(\frac{1}{g_{4}} S_{4}^{2}+\frac{1}{\gamma_{v_{4}}} \widetilde{v}_{4}^{2}\right) .
$$

The time derivative of $V_{4}$ is

$$
\begin{aligned}
\dot{V}_{4}= & S_{4}\left[\frac{\Psi_{2}}{g_{4}}\left(-\frac{\dot{\omega}_{2}}{\omega_{2}} e_{2}-\dot{y}_{r 2}\right)+\frac{\Psi_{2} f_{4}\left(\bar{x}_{4}\right)}{g_{4}}+\Psi_{2} u_{B}^{\prime}\right] \\
& +\frac{1}{\gamma_{v_{4}}} \widetilde{v}_{4} \dot{\hat{v}}_{4} .
\end{aligned}
$$

We use neural network to approximate $f_{4}\left(\bar{x}_{4}\right)$ :

$$
\frac{1}{g_{4}} \Psi_{2}\left[f_{4}\left(\bar{x}_{4}\right)-\frac{\dot{\omega}_{2}}{\omega_{2}} e_{2}-\dot{y}_{r 2}\right]=\theta_{\delta_{4}}^{* T} \psi_{4}\left(\xi_{4}\right)+\delta_{4}^{*}\left(\xi_{4}\right),
$$

where $\xi_{4}:=\left(x_{4}, \omega_{2}, \dot{\omega}_{2}, e_{2}, \dot{y}_{r 2}\right) \in \Omega_{\xi_{4}} \subset \mathbb{R}^{5}$; let $\widetilde{v}_{4}=\widehat{v}_{4}-v_{4}^{*}$, $\gamma_{v_{3}}$ is a positive design parameter, and $\widehat{v}_{4}$ is an estimate value of $v_{4}^{*}=\left\|\theta_{\delta_{4}}^{* T}\right\|^{2}$ :

$$
\begin{aligned}
\dot{V}_{4} \leq & S_{4}\left[\frac{\alpha_{4}^{2} S_{4} v_{4}^{*} \psi_{4}^{T} \psi_{4}}{2}+\Psi_{2} u_{B}^{\prime}+\frac{1}{2} S_{4}\right] \\
& +\frac{1}{2} \delta_{4}^{* 2}\left(\xi_{4}\right)+\frac{1}{2 \alpha_{4}^{2}}+\frac{1}{\gamma_{v_{4}}} \widetilde{v}_{4} \dot{\widehat{v}}_{4} .
\end{aligned}
$$

Design voltage control law and parameter adjustment law are, respectively, as follows:

$$
\begin{gathered}
u_{B}^{\prime}=\frac{1}{\Psi_{2}}\left[-k_{4} S_{4}-\frac{1}{2} S_{4}-\frac{\alpha_{4}^{2} S_{4} \widehat{v}_{4} \psi_{4}^{T} \psi_{4}}{2}\right], \\
\dot{\hat{v}}_{4}=\gamma_{v_{4}}\left[\frac{\alpha_{4}^{2} S_{4}^{2} \psi_{4}^{T} \psi_{4}}{2}-\sigma_{v_{4}} \widehat{v}_{4}\right] .
\end{gathered}
$$

Remark 7. We emphasize our dynamic surface control scheme which, combined with tracking error transformed function (15), is also suitable for the half-car active suspension system that is described in $[23,24]$. 


\section{Stability Analysis}

This section will conduct stability analysis for dynamic surface control scheme on the generator excitation system. Although the control law is simple, the stability analysis is relatively complex due to the introduction of first-order lowpass filters. For the first subsystem in (7), to begin with, we define

$$
\begin{aligned}
y_{e 2} & =z_{2}-x_{2 d} \\
& =z_{2}+\frac{\left[k_{1} S_{1}+\Psi\left(-(\dot{\omega} / \omega) e+\Psi S_{1}-\dot{y}_{r}\right)\right]}{\Psi}, \\
y_{e 3} & =z_{3}-x_{3 d}=z_{3} \pm\left(k_{2} S_{2}+\frac{\alpha_{2}^{2} S_{2} \widehat{v}_{2} \psi_{2}^{T} \psi_{2}}{2}\right),
\end{aligned}
$$

where $x_{2 d}$ and $x_{3 d}$ are designed in (22) and (31), respectively. From (23) and (33), we have

$$
\begin{aligned}
& \dot{z}_{2}=\frac{\left(x_{2 d}-z_{2}\right)}{\tau_{2}}=-\frac{y_{e 2}}{\tau_{2}}, \\
& \dot{z}_{3}=\frac{\left(x_{3 d}-z_{3}\right)}{\tau_{3}}=-\frac{y_{e 3}}{\tau_{3}} .
\end{aligned}
$$

Then, the time derivative of (47) and (48) is

$$
\begin{aligned}
\dot{y}_{e 2}= & -\frac{y_{e 2}}{\tau_{2}}+\frac{k_{1} \dot{S}_{1}}{\Psi}+\frac{k_{1} S_{1} \dot{\Psi}}{\Psi^{2}}+\left(\frac{\ddot{\omega}}{\omega} e-\frac{\dot{\omega}^{2}}{\omega^{2}} e+\frac{\dot{\omega}}{\omega} \dot{e}\right) \\
& +\left(\dot{\Psi} S_{1}+\Psi \dot{S}_{1}\right)-\ddot{y}_{r} \\
= & -\frac{y_{e 2}}{\tau_{2}}+B_{2}\left(S_{1}, S_{2}, y_{2}, \omega, \dot{\omega}, \ddot{\omega}, y_{r}, \dot{y}_{r}, \ddot{y}_{r}\right), \\
\dot{y}_{e 3}= & -\frac{y_{e 3}}{\tau_{3}} \pm\left[k_{2} \dot{S}_{2}+\frac{\alpha_{2}^{2} \dot{S}_{2} \widehat{v}_{2} \psi_{2}^{T} \psi_{2}}{2}+\frac{\alpha_{2}^{2} S_{2} \dot{\widehat{v}}_{2} \psi_{2}^{T} \psi_{2}}{2}\right. \\
& \left.+\alpha_{2}^{2} S_{2} \dot{\hat{v}}_{2} \psi_{2} \times\left(\sum_{j=1}^{2} \frac{\partial \psi_{2}}{\partial x_{j}} \dot{x}_{j}+\frac{\partial \psi_{2}}{\partial S_{2}}\right)\right] \\
= & -\frac{y_{e 3}}{\tau_{3}}+B_{3}\left(S_{1}, S_{2}, S_{3}, y_{2}, y_{3}, \widehat{v}_{2}, \omega, \dot{\omega}, \ddot{\omega}, y_{r}, \dot{y}_{r}, \ddot{y}_{r}\right),
\end{aligned}
$$

where $B_{2}$ and $B_{3}$ are continuous functions.

Theorem 8. Consider the closed-loop control system composed by object (7), first-order low-pass filter (23), (33), the actual control law (39), and parameter adjustment law (40):

$$
V=V_{1}+V_{2}+V_{3}+\frac{1}{2} y_{e 2}^{2}+\frac{1}{2} y_{e 3}^{3},
$$

where $V_{1}, V_{2}$, and $V_{3}$, respectively, are defined by (20), (25), and (35). We suppose that for the given positive constant $\delta_{i}^{*},(27)$, (37), and (44) meet $\left|\delta_{i}\left(\xi_{i}\right)\right| \leq \delta_{i}^{*}, i=2,3,4$, in a compact set. For any given positive parameter $p$, if

$$
V(0) \leq p
$$

then through the proper selection of the design parameters $k_{1}$, $k_{2}, k_{3}, \gamma_{v_{2}}, \gamma_{v_{3}}, \sigma_{v_{2}}$, and $\sigma_{v_{3}}$ all the variables in the closed-loop system are uniformly ultimately bounded. The tracking performance of the system can be specified in advance.

Proof. The derivative of $V$ by considering (51) is

$$
\dot{V}=\dot{V}_{1}+\dot{V}_{2}+\dot{V}_{3}+y_{e 2} \dot{y}_{e 2}+y_{e 3} \dot{y}_{e 3} \text {. }
$$

From (24) and (47), we have

$$
x_{2}=S_{2}+y_{e 2}+x_{2 d} .
$$

And consider the inequality

$$
\begin{aligned}
\Psi S_{1} S_{2} & \leq \frac{1}{2} \Psi^{2} S_{1}^{2}+\frac{1}{2} S_{2}^{2}, \\
\Psi S_{1} y_{e 2} & \leq \frac{1}{2} \Psi^{2} S_{1}^{2}+\frac{1}{2} y_{e 2}^{2} .
\end{aligned}
$$

Substituting (54) and (55) and virtual control law (22) into (21), we have

$$
\dot{V}_{1} \leq-k_{1} S_{1}^{2}+\frac{1}{2} S_{2}^{2}+\frac{1}{2} y_{e 2}^{2} .
$$

Using (34) and (48),

$$
x_{3}=S_{3}+y_{e 3}+x_{3 d} .
$$

At the same time,

$$
\begin{gathered}
g_{2} S_{2} S_{3} \leq \frac{1}{2} g_{2}^{2} S_{2}^{2}+\frac{1}{2} S_{3}^{2}, \\
g_{2} S_{2} y_{e 3} \leq \frac{1}{2} g_{2}^{2} S_{2}^{2}+\frac{1}{2} y_{e 3}^{2} .
\end{gathered}
$$

Substitute (57) and (58), virtual control law (31), and parameter adjustment law (32) into (30):

$$
\begin{aligned}
\dot{V}_{2} \leq & -k_{2}\left|g_{2}\right| S_{2}^{2}+\frac{1}{2} S_{3}^{2}+\frac{1}{2} y_{e 3}^{2} \\
& +\frac{1}{2} \delta_{2}^{* 2}+\frac{g_{2 \max }^{2}}{2 \alpha_{2}^{2}}-g_{m} \sigma_{v_{2}} \widehat{v}_{2} \widetilde{v}_{2} .
\end{aligned}
$$

From (39) and (40), (38) can be transformed as

$$
\dot{V}_{3} \leq-k_{3} S_{3}^{2}+\frac{1}{2} \delta_{3}^{* 2}+\frac{1}{2 \alpha_{3}^{2}}-\sigma_{v_{3}} \widehat{v}_{3} \widetilde{v}_{3} .
$$

According to Assumption 4,

$$
\Pi:=\left\{\left(y_{r}, \dot{y}_{r}, \ddot{y}_{r}\right): y_{r}^{2}+\dot{y}_{r}^{2}+\ddot{y}_{r} \leq B_{0}\right\}
$$

is a compact set in $\mathbb{R}^{3}$, and $B_{0}>0 . M_{2}$ is the maximum of $\left|B_{2}\right|$ in $\Pi ; M_{3}$ is the maximum of $\left|B_{3}\right|$ at $\Pi$. For any $p>0$, using inequality, we can obtain that

$$
\begin{aligned}
& \left|y_{e 2} B_{2}\right| \leq \frac{y_{e 2}^{2} B_{2}^{2}}{2 \mu}+\frac{\mu}{2} \leq \frac{y_{e 2}^{2} M_{2}^{2}}{2 \mu}+\frac{\mu}{2}, \\
& \left|y_{e 3} B_{3}\right| \leq \frac{y_{e 3}^{2} B_{3}^{2}}{2 \mu}+\frac{\mu}{2} \leq \frac{y_{e 3}^{2} M_{3}^{2}}{2 \mu}+\frac{\mu}{2},
\end{aligned}
$$


where $\mu$ is a positive constant parameter

$$
\begin{aligned}
& \frac{1}{\tau_{2}}=\frac{1}{2}+\frac{M_{2}^{2}}{2 \mu}+\alpha_{0}, \\
& \frac{1}{\tau_{3}}=\frac{1}{2}+\frac{M_{3}^{2}}{2 \mu}+\alpha_{0} .
\end{aligned}
$$

Furthermore,

$$
\begin{gathered}
-g_{m} \sigma_{v_{2}} \widehat{v}_{2} \widetilde{v}_{2} \leq-\frac{\sigma_{v_{2}} g_{m}}{2} \widetilde{v}_{2}^{2}+\frac{\sigma_{v_{2}} g_{m}}{2} v_{2}^{* 2}, \\
-\sigma_{v_{3}} \widehat{v}_{3} \widetilde{v}_{3} \leq-\frac{\sigma_{v_{3}}}{2} \widetilde{v}_{3}^{2}+\frac{\sigma_{v_{3}}}{2} v_{3}^{* 2} .
\end{gathered}
$$

Taking (56), (59), (60), and (62)-(66) into consideration, (53) can be transformed into the following equation:

$$
\begin{aligned}
\dot{V} \leq & -k_{1} S_{1}^{2}-\left(k_{2} g_{m}-\frac{1}{2}\right) S_{2}^{2}-\left(k_{3}-\frac{1}{2}\right) S_{3}^{2} \\
& -\alpha_{0} y_{e 2}^{2}-\alpha_{0} y_{e 3}^{2}-\frac{\sigma_{v_{2}} g_{m}}{2} \widetilde{v}_{2}^{2}-\frac{\sigma_{v_{3}}}{2} \widetilde{v}_{3}^{2}+C,
\end{aligned}
$$

where

$$
\begin{aligned}
C= & \frac{\sigma_{v_{2}} g_{m}}{2} v_{2}^{* 2}+\frac{\sigma_{v_{3}}}{2} v_{3}^{* 2}+\mu+\frac{1}{2} \delta_{2}^{* 2} \\
& +\frac{1}{2} \delta_{3}^{* 2}+\frac{g_{2 \max }^{2}}{2 \alpha_{2}^{2}}+\frac{1}{2 \alpha_{3}^{2}} .
\end{aligned}
$$

And $\alpha_{0}$ is a positive design parameter:

$$
\alpha_{0} \leq \min \left\{k_{1}, k_{2} g_{m}-\frac{1}{2}, k_{3}-\frac{1}{2}, \frac{\gamma_{v_{2}} \sigma_{v_{2}}}{2}, \frac{\gamma_{v_{3}} \sigma_{v_{3}}}{2}\right\} \text {. }
$$

From (67), we have

$$
\dot{V} \leq-2 \alpha_{0} V+C
$$

Let

$$
\alpha_{0}>\frac{C}{2 p}
$$

We can see that when $V=p, \dot{V} \leq 0$. So, $V \leq p$ is an invariant set. If $V(0) \leq p$, then, for all $t \geq 0, V(t) \leq p$. From (70), $\forall t \geq 0$,

$$
0 \leq V(t) \leq \frac{C}{2 \alpha_{0}}+\left\{V(0)-\frac{C}{2 \alpha_{0}}\right\} e^{-2 \alpha_{0} t} .
$$

Then,

$$
\lim _{t \rightarrow \infty} V(t)=\frac{C}{2 \alpha_{0}} .
$$

Similar to the second subsystems, we substitute (46) into (45):

$$
\dot{V}_{4} \leq-k_{4} S_{4}^{2}-\frac{\sigma_{v_{4}}}{2} \widetilde{v}_{4}^{2}+\frac{1}{2} \delta_{4}^{* 2}+\frac{1}{2 \alpha_{4}^{2}}+\frac{\sigma_{v_{4}}}{2} v_{4}^{* 2} .
$$

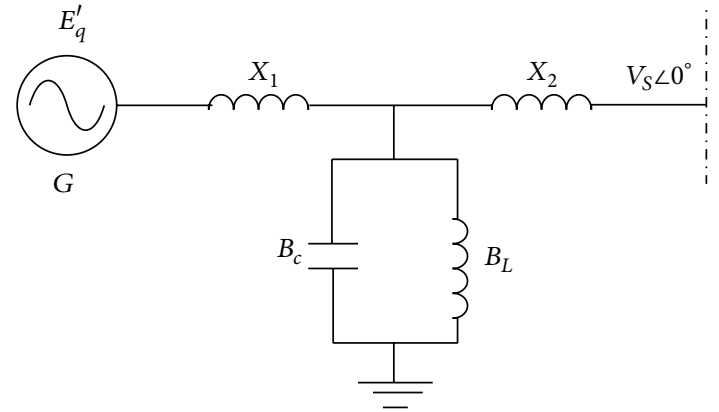

FIgURE 2: The equivalent circuit diagram.

Let

$$
\begin{gathered}
k_{4} \geq \frac{a}{g_{4}}, \\
\sigma_{v_{4}} \geq \frac{2 a}{\gamma_{v_{4}}}, \\
C_{2}=\frac{\sigma_{v_{4}}}{2} v_{4}^{* 2}+\frac{1}{2} \delta_{4}^{* 2}+\frac{1}{2 \alpha_{4}^{2}} .
\end{gathered}
$$

We have

$$
\begin{aligned}
& \dot{V}_{4} \leq-2 a V+C_{2}, \\
& \lim _{t \rightarrow \infty} V_{4}(t)=\frac{C}{2 a} .
\end{aligned}
$$

As a result, all the signals such as $S_{1}, S_{2}, S_{3}, S_{4}, k_{1}, k_{2}$, $k_{3}, k_{4}, \sigma_{v_{2}}, \sigma_{v_{3}}, \sigma_{v_{4}}, \alpha_{0}$, and $a$ in the closed-loop system are uniformly bounded. In addition, (69), (75), and (76) show that, by selecting appropriate design parameters, $\alpha_{0}$, a can be infinite; (73) and (79) show that the tracking error can converge to an arbitrary small value.

\section{Simulation Analysis}

The single machine infinite bus power system with SVC is shown in Figures 1 and 2, assuming that the amplitude and frequency of receiving end bus voltage are not changed.

System parameters are $X_{d}^{\prime}=0.344, X_{d}=0.982, X_{T}=0.1$, $D=2, X_{d \Sigma}^{\prime}=0.6967, B_{L}=1.55, B_{C}=0.5, T_{d 0}=5, T_{C}=2$, $H=13, E_{q}^{\prime}=1.059, \delta_{0}=40, \omega_{0}=100 \pi \mathrm{rad} / \mathrm{s}, P_{m 0}=1$, $V_{s}=1, V_{\text {ref }}=1, X_{2}=0.35$, and $X_{d \Sigma}=1.4$.

In the simulation the initial values of the states are $x_{1}(0)=$ $40, x_{2}(0)=100 \pi, x_{3}(0)=1.05$, and $x_{3}(0)=1$. For $N N$ Gaussian function $\psi_{j}\left(\xi_{i}\right)=\exp \left[-\left(\xi_{i}-\zeta_{j}\right)^{T}\left(\xi_{i}-\zeta_{j}\right) / \eta_{j}^{2}\right]$, $i=2,3,4$, we choose 21 nodes with the centers of the basis functions $\zeta_{j}$ evenly spaced in $[-40,+40] \times[-314,+3144] \times$ $[-2,+2] \times[-1,+1]$ and width $\eta_{j}=1$, for $j=1, \ldots, 21$. Also, we choose a smooth and strictly monotone increasing function, $\Phi_{2}\left(S_{4}\right)=(2 / \pi) \arctan \left(S_{4}\right), \omega(t)=0.65 e^{-8 t}+0.002$ and $S_{4}=\tan \left((\pi / 2) \cdot\left(e_{2}(t) / \bar{w}_{2}(t)\right)\right)$, which means that $\Psi_{2}=$ $(\pi / 2) \cdot\left(1 / \omega_{2}\right) \cdot \cos ^{-2}\left((\pi / 2) \cdot\left(e_{2} / \omega_{2}\right)\right)$. 


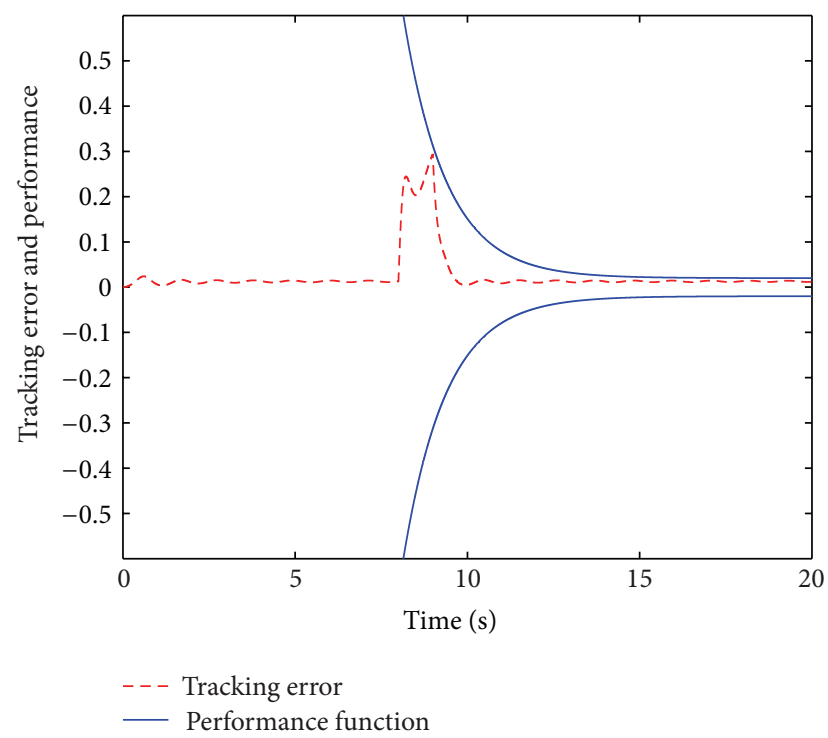

FIGURE 3: Tracking error and the performance function.

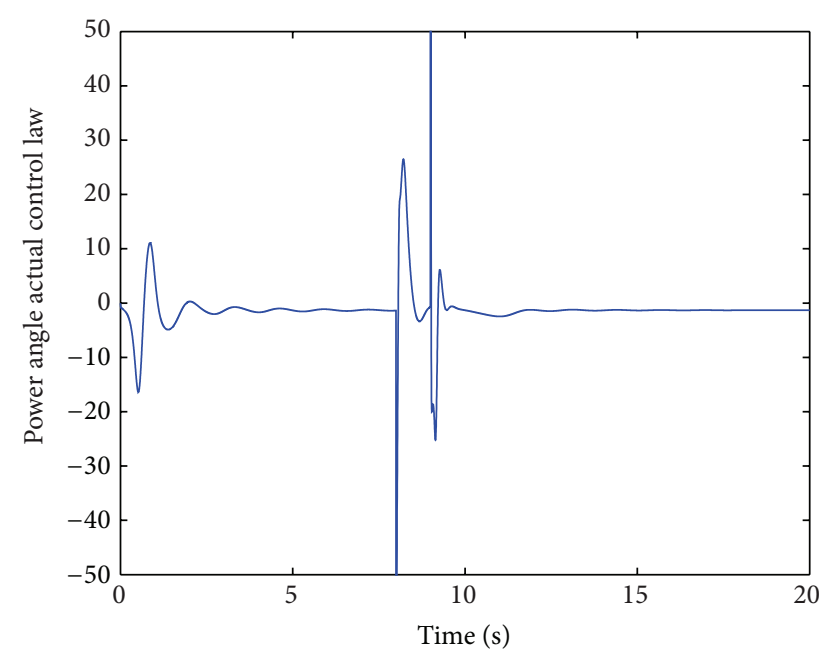

FIgURE 4: The actual control law $u_{f}$ of power angle.

We suppose that three-phase short-circuit occurred on the terminal of the line at $t=8 \mathrm{~s}$ and eliminate the fault at $t=9 \mathrm{~s}$; the simulation results are shown in Figures 3, 4, 5, 6, $7,8,9,10,11,12$, and 13 . Figure 3 shows that, by introducing the error transformed function, the system can be stabilized promptly when it encountered some emergencies such as three-phase short-circuit which means that our control scheme makes the generator excitation control system have strong robustness. The tracking error of power angle can be prespecified by using the performance function $\omega(t)=$ $0.65 e^{-8 t}+0.002$. Figures 4 and 5 are actual control of power angle and actual voltage control law of the access point voltage, respectively. From Figure 6-Figure 8 we can know that all the states in the control system recover to their desired values in a short time by using our dynamic surface control

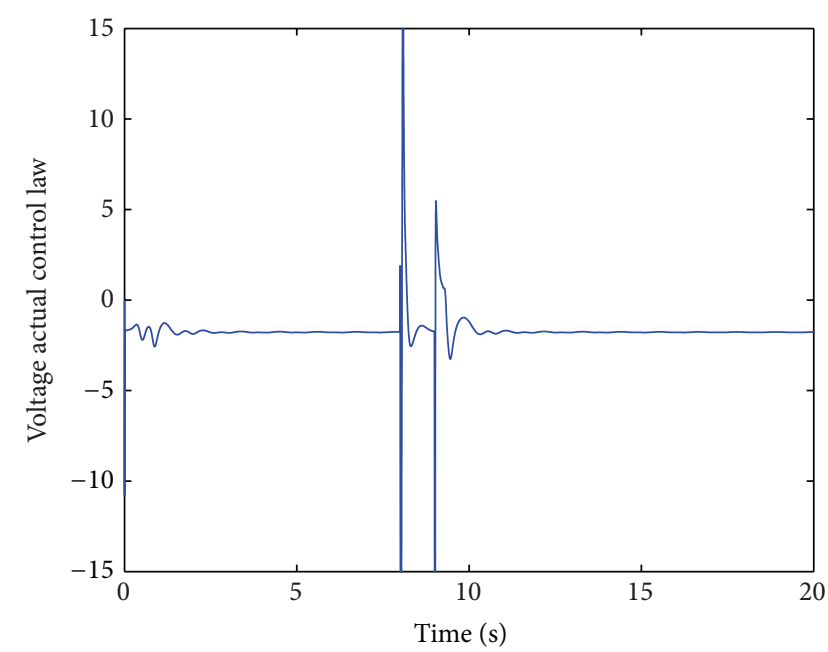

FIGURE 5: The actual voltage control law $u_{B}^{\prime}$ of access point voltage.

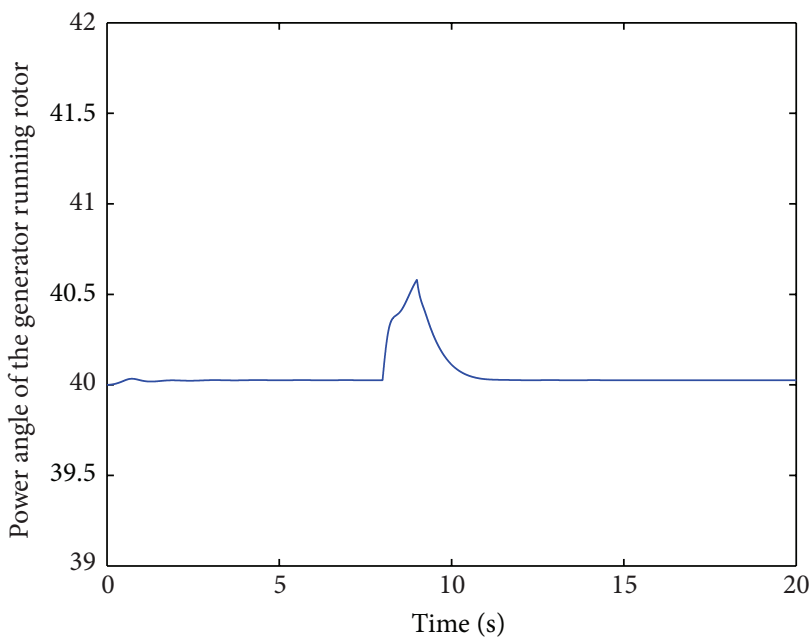

Figure 6: The rotor angle $\delta$ of generator.

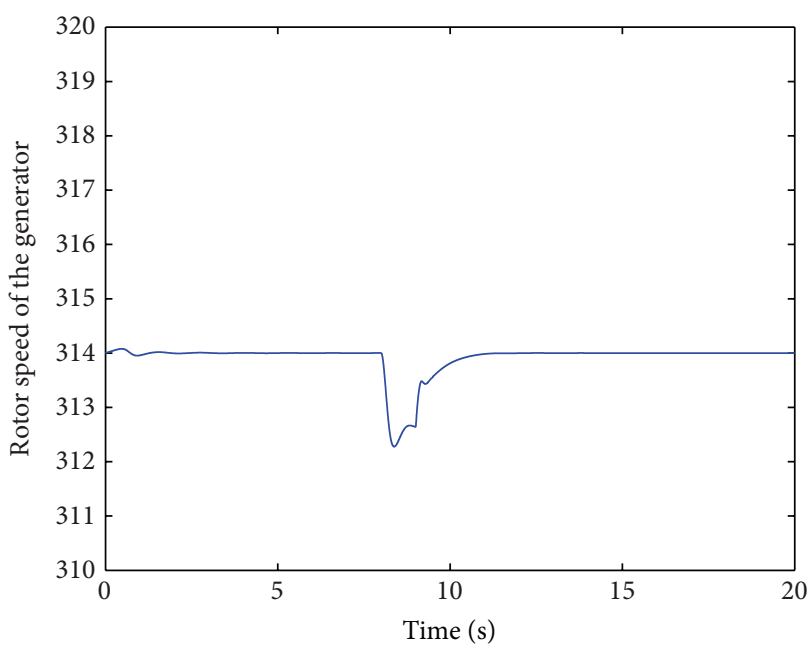

FIGURE 7: The rotor speed $\omega$ of generator. 


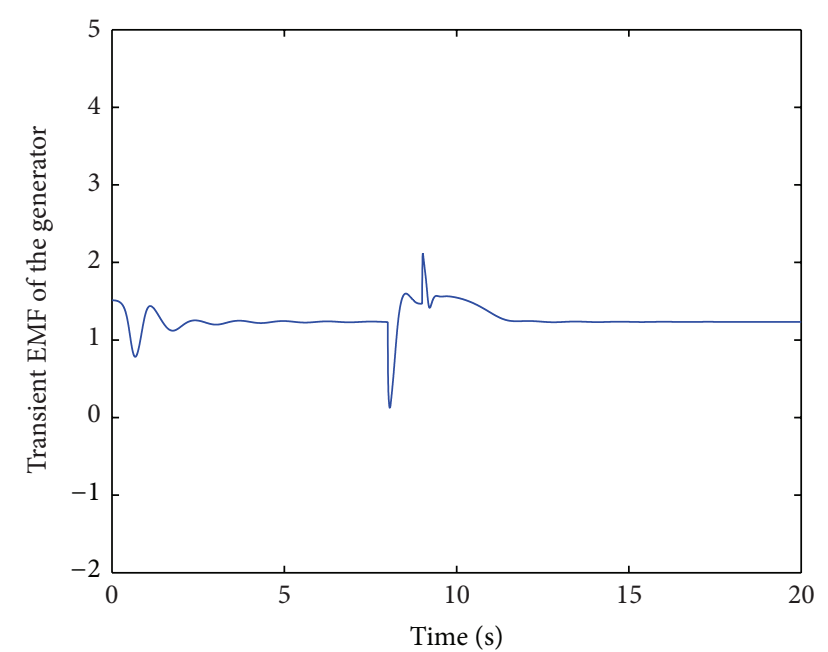

FIgURE 8: The transient EMF $E_{q}^{\prime}$ of the generator.

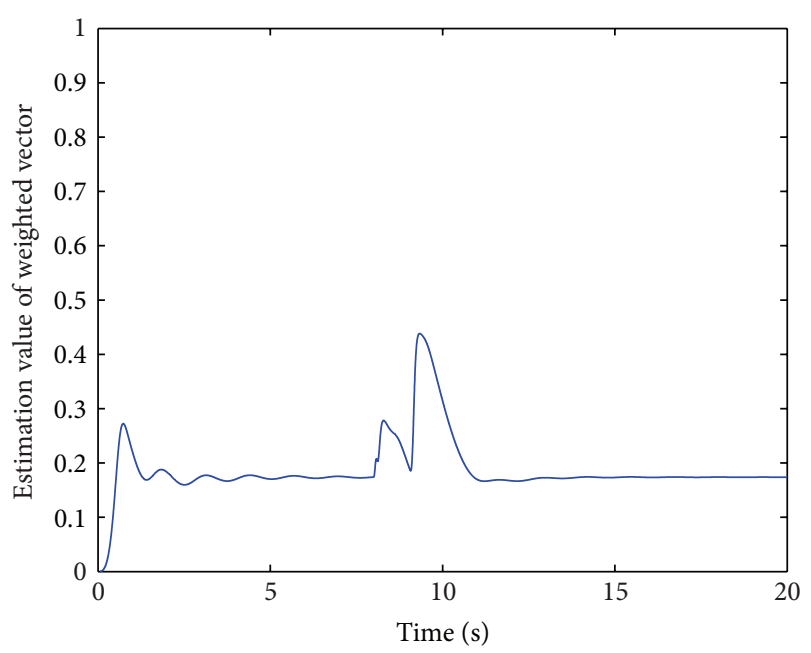

FIGURE 9: The estimated norm of the weighted vector $\widehat{v}_{2}$, with $v_{2}^{*}=$ $\left\|\theta_{\delta_{2}}^{* T}\right\|^{2}$.

scheme. Figure 9 to Figure 11 show the estimations of weight vector norm of the neural network. Figure 12 is the access point voltage increment $V_{m}-V_{\text {ref }}$, whose tracking process can also be arbitrarily prespecified by using performance function as that in Figure 3.

Remark 9. The controllers that have been proposed in $[12,13]$ are based on backstepping technique, which can cause the explosion of complicity problem that means the controller will become complex when the relative degree of the control system is high. Also, our novel neural network based adaptive dynamic surface controller is designed without doing any linearization to the excitation generator model. Therefore, the design procedures and the final control law are greatly simplified and the control accuracy is improved. Figure 13 has shown the comparison of the tracking error between our control scheme and backstepping control scheme.

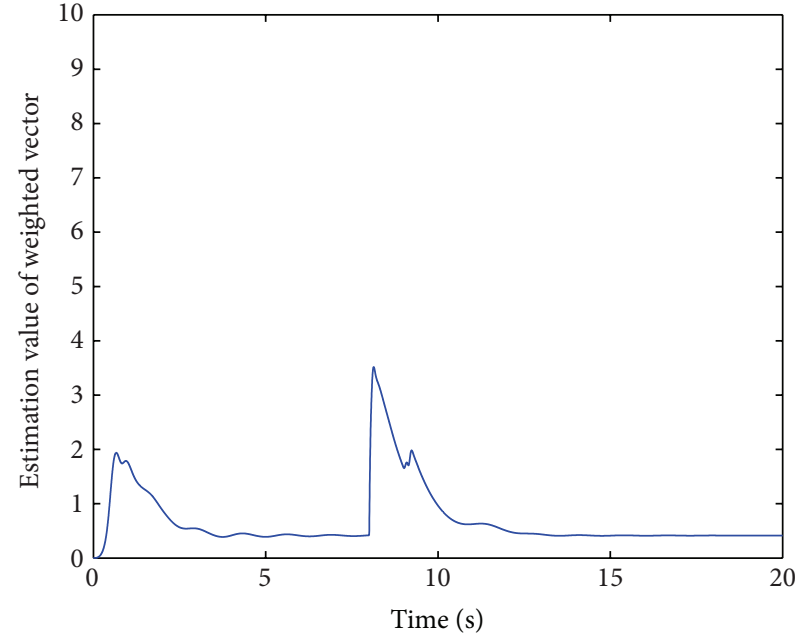

FIGURE 10: The estimated norm of the weighted vector $\widehat{v}_{3}$, with $v_{3}^{*}=$ $\left\|\theta_{\delta_{3}}^{* T}\right\|^{2}$.

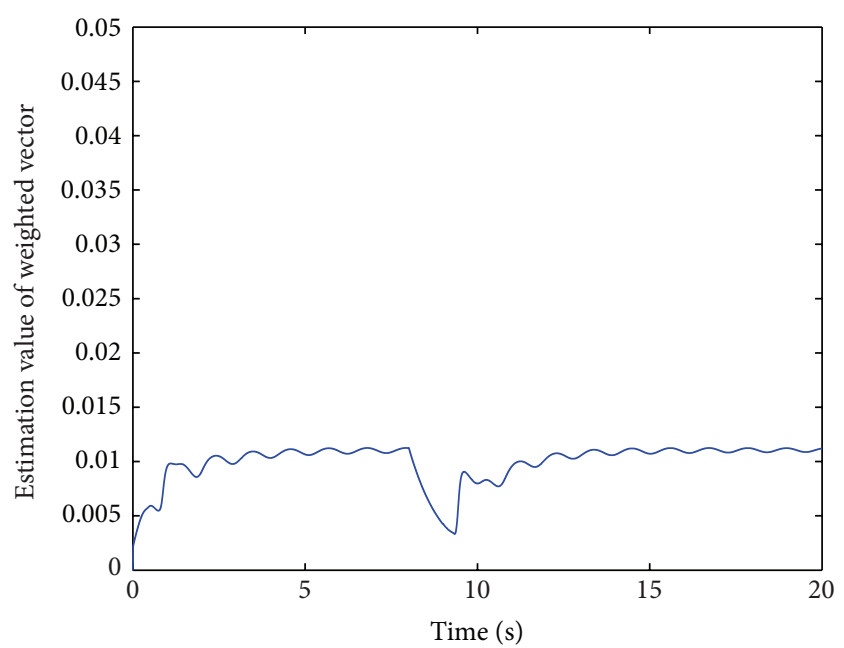

FIGURE 11: The estimated norm of the weighted vector $\widehat{v}_{4}$, with $v_{4}^{*}=$ $\left\|\theta_{\delta_{4}}^{* T}\right\|^{2}$.

\section{Conclusion}

This paper proposed a novel neural network and tracking error transformed function based on adaptive dynamic surface control scheme for the generator excitation control system which is equipped with SVC. In this scheme, both the designing procedures and the control law are simplified because it does not need to make any transformation to the excitation generator model. The prespecified tracking performance can be guaranteed by using the tracking error transformed function. The norm of the weighted vector of neural network is estimated which replaces the estimation of the weighted vector itself; therefore, the computational burden is greatly reduced. Also, the explosion of complicity problem inherent in the backstepping control can be eliminated. It is proved that the new scheme can make the system semiglobally uniformly ultimately bounded. 


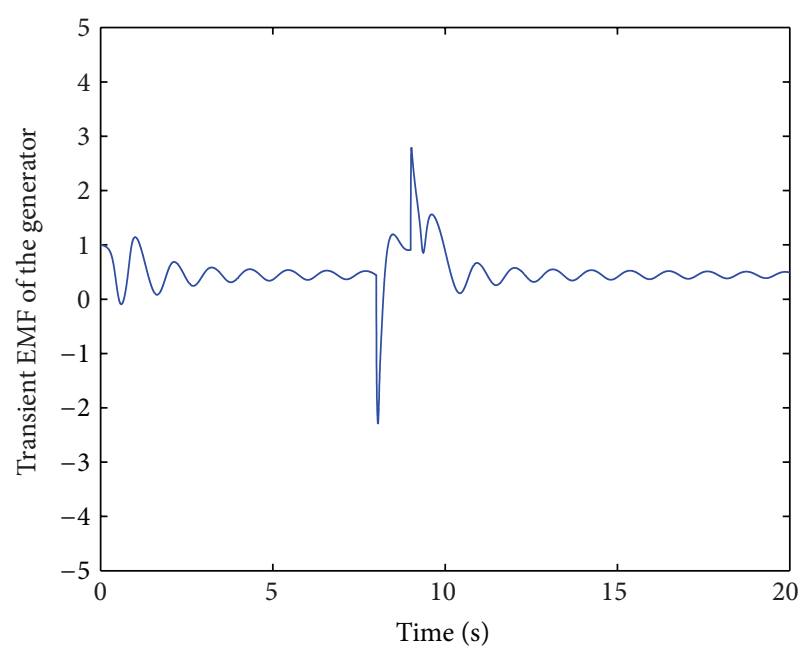

FIGURE 12: The access point voltage increment $V_{m}-V_{\text {ref }}$.

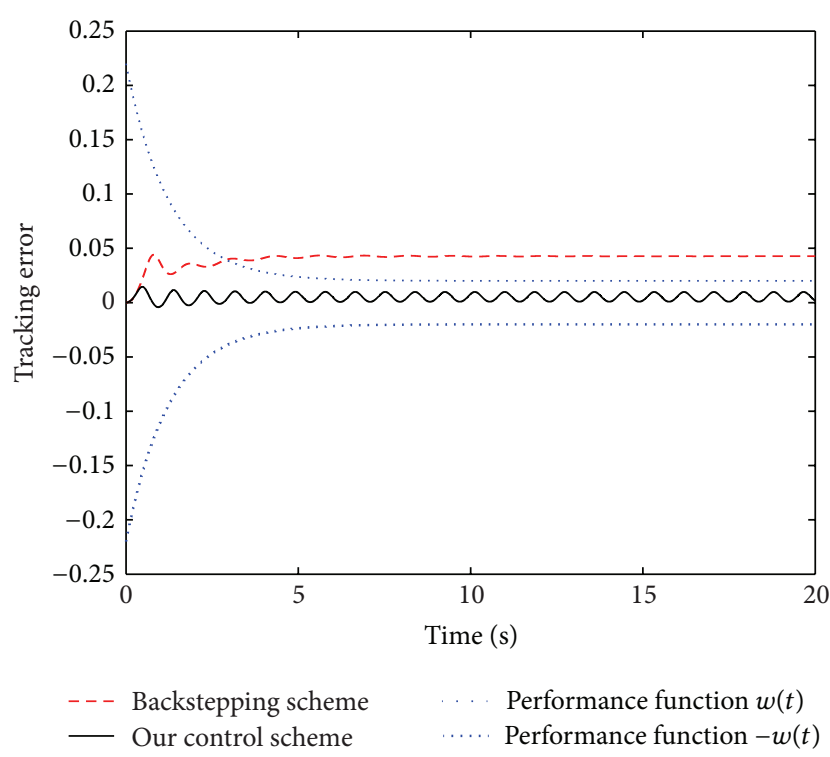

FIGURE 13: The comparison of our NN based dynamic surface control scheme and backstepping.

\section{Conflict of Interests}

The authors declare that there is no conflict of interests regarding the publication of this paper.

\section{Acknowledgments}

The research is supported by Natural Science Foundation (no. 61304015), China Postdoctoral Science Foundation (no. 2013M540839), Outstanding Young Scholar Project of Jilin City (no. 2013625002), “Twelfth Five Year” Scientific Research Plan of Jilin Province (no. [2014]111), and Nature Science Foundation of Jilin Province (no. 20140101059).

\section{References}

[1] P. Kundur, Power System Stability and Control, China Electric Power Press, Beijing, China, 2002.

[2] A. J. Saavedra-Montes, J. M. Ramirez-Scarpetta, and O. P. Malik, "Methodology to estimate parameters of an excitation system based on experimental conditions," Electric Power Systems Research, vol. 81, no. 1, pp. 170-176, 2011.

[3] F. Zhu, H. Zhao, Z. Liu et al., "Effects of interconnected power grid on power system dynamic stability," Proceedings of the CSEE, vol. 27, no. 1, pp. 1-7.

[4] W. Ben and M. Zongyuan, "Giant synchronous generation-set's variable structure controller," Proceedings of the EPSA, vol. 10, no. 2, pp. 1-6, 1998.

[5] Y. Peihong, "The influence of excitation system to the stable operation of the power grid," Inner Mongolia University of Science and Technology, Inner Mongolia, China, 2008.

[6] H. Renmu, S. Feng, H. Dong et al., "The study of generator excitation system modeling and parameters estimation," Power System Technology, vol. 31, no. 14, pp. 62-67, 2007.

[7] Q. Liu, The Stability of Powersystem and Excitation Control System, China Electric Power Press, Beijing, China, 2007.

[8] K. Fregene and D. Kennedy, "Stabilizing control of a highorder generator model by adaptive feedback linearization," IEEE Transactions on Energy Conversion, vol. 18, no. 1, pp. 149-156, 2003.

[9] G. Fusco and M. Russo, "A nonlinear control of synchronous generator excitation for voltage regulation in power systems," in Proceedings of the Mediterranean Conference on Control and Automation (MED '08), pp. 158-162, Ajaccio, France, June 2008.

[10] A. Colbia-Vega, J. de León-Morales, L. Fridman, O. Salas-Peña, and M. T. Mata-Jiménez, "Robust excitation control design using sliding-mode technique for multimachine power systems," Electric Power Systems Research, vol. 78, no. 9, pp. 16271634, 2008.

[11] L. Wan and Y. Chen, "Comprehensive nonlinear robust control for steam-valving and excitation system applying zero dynamic and variable structure control," Proceedings of the Chinese Society of Electrical Engineering, vol. 28, no. 13, pp. 26-32, 2008.

[12] H. A. Yousef, M. Hamdy, and M. Shafiq, "Flatness-based adaptive fuzzy output tracking excitation control for power system generators," Journal of the Franklin Institute, vol. 350, no. 8, pp. 2334-2353, 2013.

[13] F. Zhang and S. Zhu, "Adaptive excitation controller based on strong tracking filter," Proceedings of the Chinese Society of Electrical Engineering, vol. 25, no. 23, pp. 31-35, 2005.

[14] S. Mehraeen, S. Jagannathan, and M. L. Crow, "Power system stabilization using adaptive neural network-based dynamic surface control," IEEE Transactions on Power Systems, vol. 26, no. 2, pp. 669-680, 2011.

[15] X. Wang, Adaptive Variable Structure Control of Nonlinear Power System, Nanjing University of Science and Technology, Nanjing, China, 2011.

[16] L. Xiong, M. Xu, and S. Yuanzhang, "Nonlinear constant reactive power excitation control," Electric Power Automation Equipment, vol. 24, no. 5, pp. 1-4, 2004.

[17] Y. Wei, W. Wensheng, and H. Zhiming, "The Ddirect feedback linearization method for designing SSSC nonlinear control law," Proceedings of the Chinese Society of Electrical Engineering, vol. 23, no. 3, pp. 65-68, 2003. 
[18] S. Zhou and X. Wang, "Direct feedback linearization based nonlinear excitation controller," Proceedings of the Chinese Society of Electrical Engineering, vol. 15, no. 4, pp. 281-288, 1995.

[19] B. Ren, S. S. Ge, C. Su, and T. H. Lee, "Adaptive neural control for a class of uncertain nonlinear systems in pure-feedback form with hysteresis input," IEEE Transactions on Systems, Man, and Cybernetics B: Cybernetics, vol. 39, no. 2, pp. 431-443, 2009.

[20] R. Sanne M and M. J. Mears, "Stable adaptive tracking of uncertainty systems using nonlinearly parameterized on-Line approximators," IEEE Transactions on Neural Networks, vol. 3, no. 6, pp. 837-863, 1992.

[21] C. P. Bechlioulis and G. A. Rovithakis, "Adaptive control with guaranteed transient and steady state tracking error bounds for strict feedback systems," Automatica, vol. 45, no. 2, pp. 532-538, 2009.

[22] C. P. Bechlioulis and G. A. Rovithakis, "Robust adaptive control of feedback linearizable MIMO nonlinear systems with prescribed performance," IEEE Transactions on Automatic Control, vol. 53, no. 9, pp. 2090-2099, 2008.

[23] W. Sun, H. Gao, and O. Kaynak, "Adaptive backstepping control for active suspension systems with hard constraints," IEEE/ASME Transactions on Mechatronics, vol. 18, no. 3, pp. 1072-1079, 2013.

[24] W. Sun, Z. Zhao, and H. Gao, "Saturated adaptive robust control for active suspension systems," IEEE Transactions on Industrial Electronics, vol. 60, no. 9, pp. 3889-3896, 2013. 


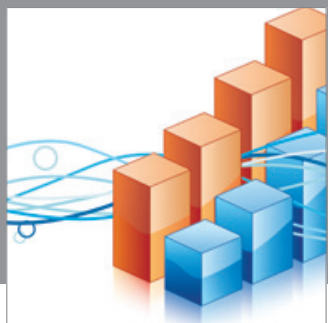

Advances in

Operations Research

mansans

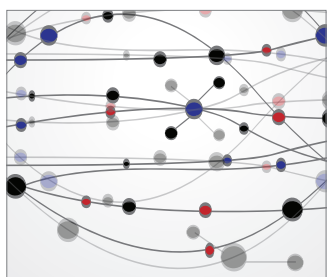

The Scientific World Journal
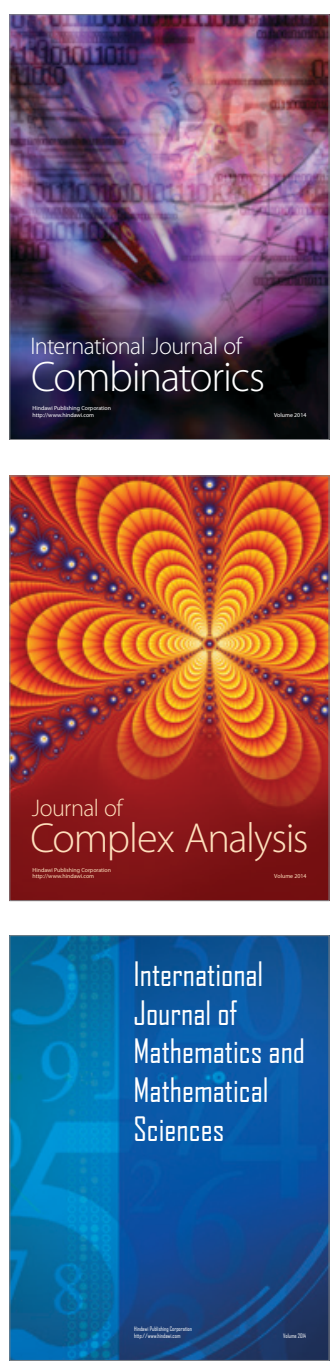
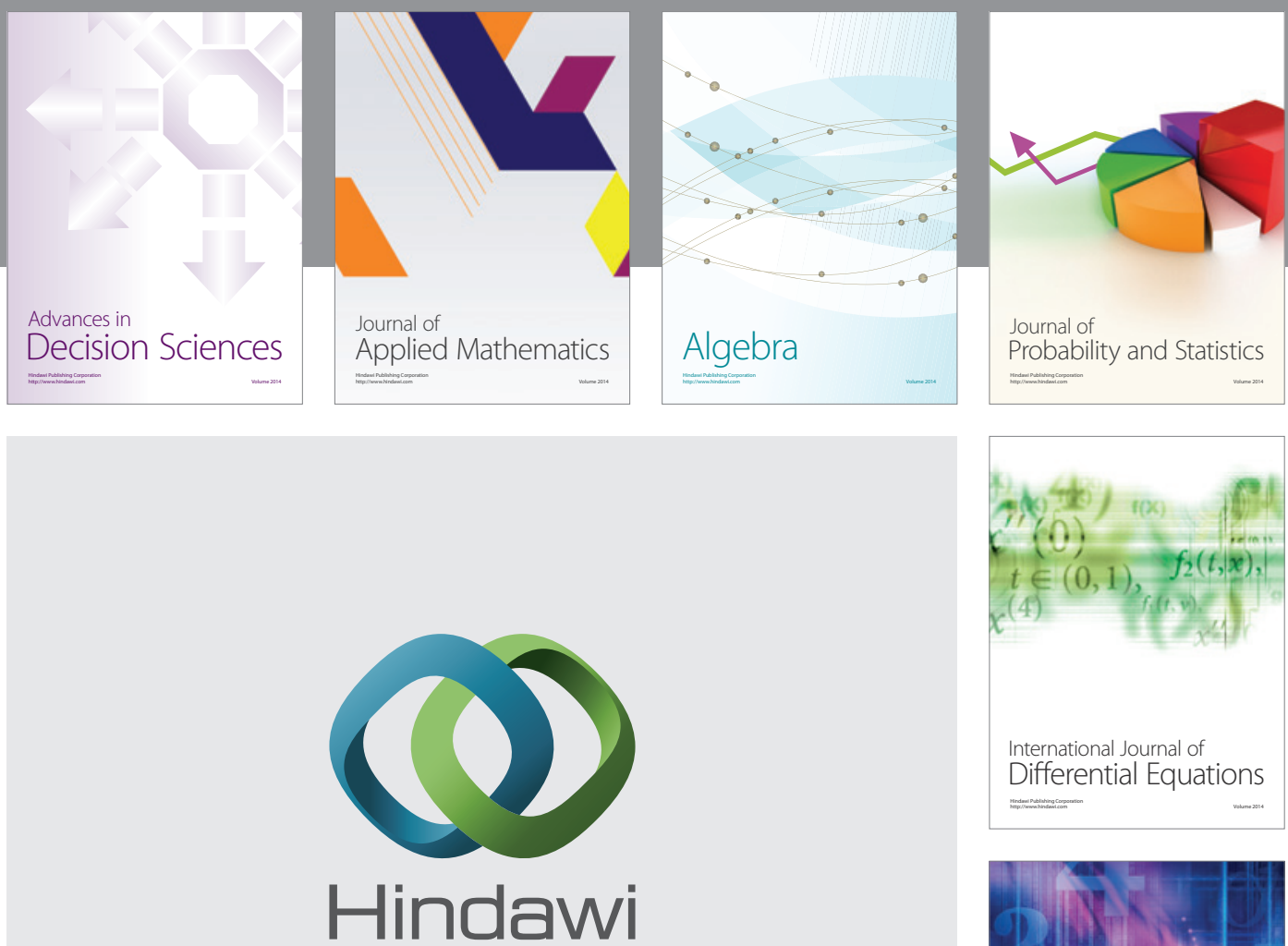

Submit your manuscripts at http://www.hindawi.com
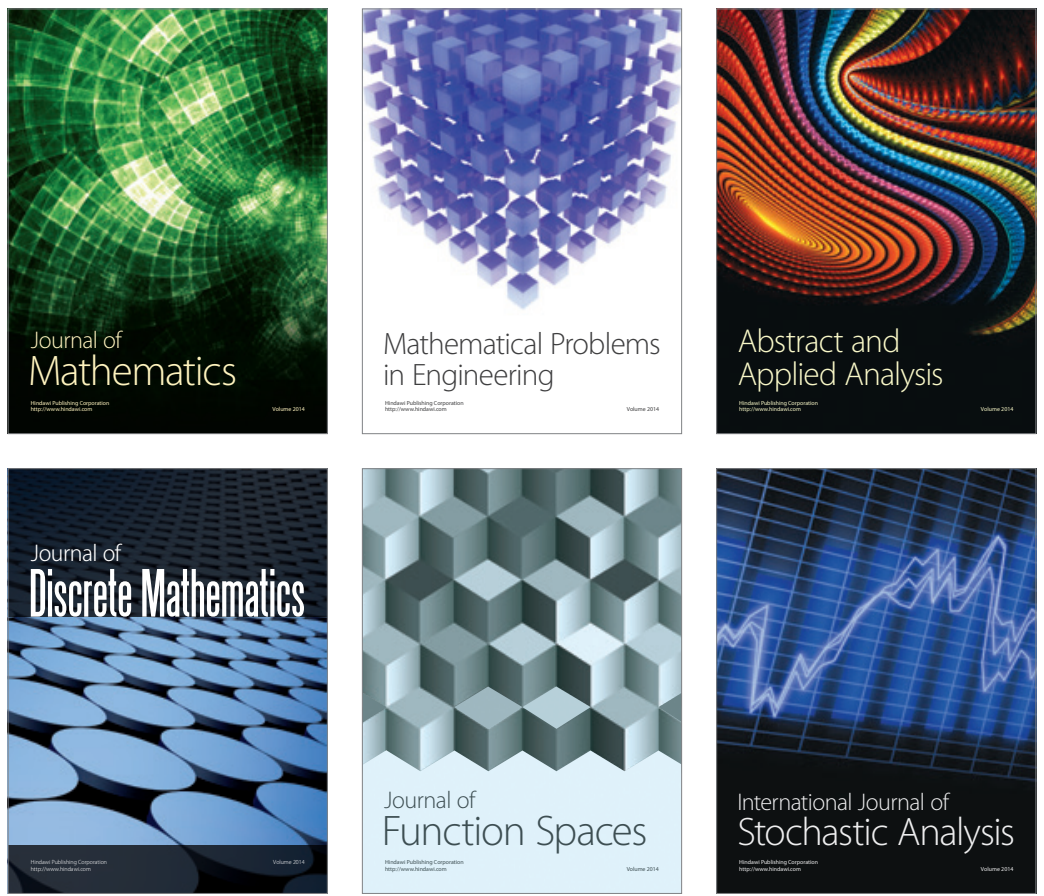

Journal of

Function Spaces

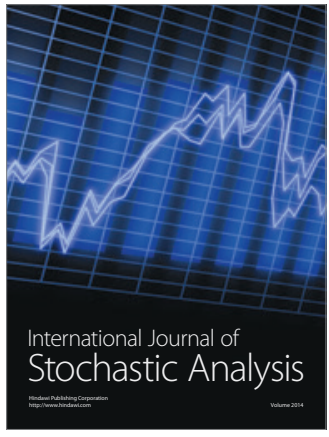

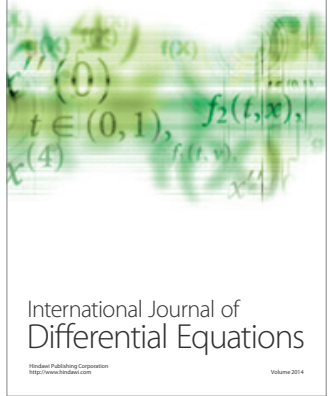
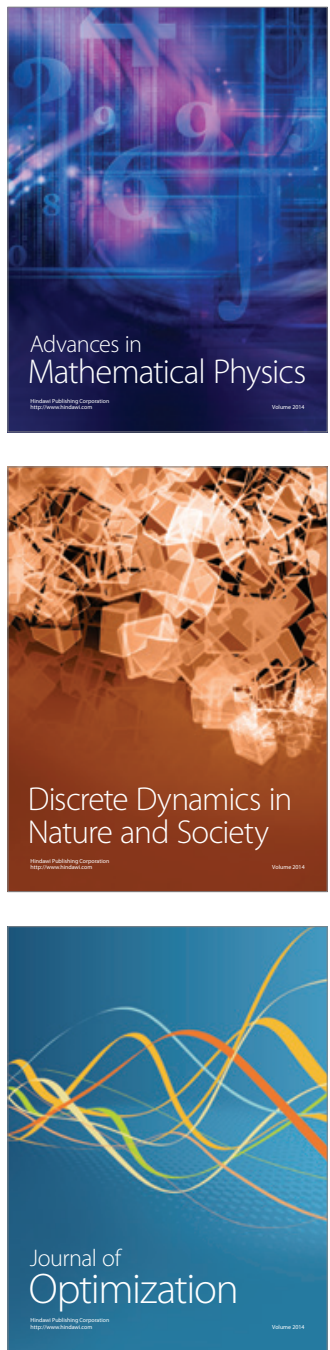